\title{
La adquisición de vocabulario en una lengua extranjera: de la teoría a la aplicación didáctica
}

\author{
ULRIKE OSTER \\ Facultad de Ciencias Humanas y Sociales \\ Universitat Jaume I
}

Recibido: 13 noviembre 2007 / Aceptado: 17 marzo 2008

ISSN: $1697-7467$

\begin{abstract}
RESUMEN: Este artículo tiene como objetivo resumir algunos fundamentos de la adquisición de vocabulario procedentes de disciplinas varias como la psicología cognitiva y la lingüística de corpus, así como analizar las repercusiones de estos conocimientos sobre el proceso de enseñanza-aprendizaje. Los enfoques presentados convergen en la idea de la interrelación y dependencia mutua de las unidades léxicas, tanto en el léxico mental como en el nivel del uso, por lo que la última parte del trabajo está dedicada a ilustrar esta cuestión con un ejemplo de actividad didáctica basada en corpus que retoma y aplica varios de los aspectos descritos.

Palabras clave: Adquisición de vocabulario, enseñanza de lenguas extranjeras, competencia léxica, uso didáctico de corpus electrónicos.
\end{abstract}

Vocabulary Acquisition in a Foreign Language: From a Theoretical Framework to an Actual Implementation.

\begin{abstract}
This paper sets out with a brief overview on findings from different research areas such as cognitive psychology and corpus linguistics that are relevant to the understanding of vocabulary acquisition, reaching the conclusion that the relatedness of lexical units is a crucial aspect both for their meaning in the mental lexicon and on the level of usage. It then analyses how these findings can be applied to the development of lexical competence. The last part of the paper illustrates the application of several of the issues described in the previous sections through a vocabulary-oriented task involving the use of corpora.

Key words: vocabulary acquisition, foreign language teaching, lexical competence, data-driven learning.
\end{abstract}

\section{INTRODUCCIÓN}

Tener un vocabulario amplio es esencial para la comprensión de una lengua extranjera. Sin embargo, las frecuentes críticas de la práctica de aprender vocabulario en forma de listas como si fueran equivalencias unívocas indican claramente que no se trata únicamente de una cuestión cuantitativa sino sobre todo cualitativa. De aquí se deriva la primera pregunta que nos hemos planteado en este artículo: ¿En qué consiste «saber una palabra»? Basándonos en las 
respuestas a esta pregunta, que encontraremos en diversos estudios sobre la adquisición de lenguas, la psicología cognitiva, la lingüística de corpus, etc., abordaremos la segunda cuestión: ¿Cómo se pueden aplicar estos conocimientos en la clase de lengua extranjera con el fin de fomentar el proceso de adquisición de vocabulario? Finalmente, mostraremos un ejemplo concreto de aplicación didáctica al aprendizaje del alemán que combina varios de los aspectos descritos. ${ }^{1}$

\section{2. ¿EN QUé CONSISTE «SABER UNA PALABRA»?}

El conocimiento de una palabra incluye distintos aspectos debido a que cada unidad léxica abarca varios componentes. Por una parte está el conocimiento semántico (el significado propiamente dicho de la palabra, incluidas las connotaciones y asociaciones que esta conlleva) y por otra parte el conocimiento sobre la forma, que a su vez incluye los aspectos fonético y gráfico así como la composición de la unidad léxica de unidades más pequeñas. El tercer tipo de conocimiento sobre la unidad léxica -aquel relacionado con su uso- comprende las funciones gramaticales de la misma, sus colocaciones (las posibilidades de combinación con otras unidades léxicas) así como las limitaciones de su uso (vid. Nation 2005: 47). Estos tres componentes no son independientes entre sí sino que existen diversos puntos de encuentro: Por ejemplo, las colocaciones se aprecian ante todo en el uso de las palabras, pero también influyen sobre el significado de las mismas. Y también el hecho formal de que una unidad léxica se componga de otras unidades tiene repercusiones clarísimas sobre su significado. Esta concepción de la competencia léxica subraya, pues, la estrecha interrelación entre léxico y gramática, tal y como se está defendiendo en gran parte de las corrientes de la lingüística actual y como se ha empezado a notar también en muchas aplicaciones a la didáctica de lenguas.

De los tres tipos de conocimiento mencionados -significado, forma y uso-, los aspectos que más complejos resultan en el aprendizaje de una lengua extranjera son seguramente aquellos que están relacionados con la semántica (porque el significado en sí es complejo y porque en contraste con otra lengua lo es aún más) y con el uso. Por este motivo, en los siguientes apartados procuraremos profundizar en dos aspectos del significado de las unidades léxicas que son especialmente importantes para la adquisición del vocabulario: la representación mental del significado así como las relaciones combinatorias de unas palabras con otras.

\subsection{La representación del conocimiento semántico en el lexicón mental}

El funcionamiento del cerebro y la influencia de ello sobre el aprendizaje de lenguas es un tema complejo que ha sido y es objeto de numerosas investigaciones neurolingüísticas. Por ejemplo, la idea de una división funcional estricta entre hemisferio izquierdo y hemisferio derecho ha sido relativizada en investigaciones más recientes. Si bien es cierto que estudios realizados con pacientes afásicos aquejados de diferentes lesiones cerebrales han mostrado

\footnotetext{
${ }^{1}$ La aplicación didáctica se ha desarrollado en el marco de un proyecto de investigación financiado por el Ministerio de Educación y Ciencia sobre CIBERGENEROS Y TECNOLOGIAS APLICADAS A LA AUTONOMIA DE APRENDIZAJE DE LENGUAS (HUM2005-05548/FILO), llevado a cabo por el grupo GIAPEL, de la Universitat Jaume I de Castellón.
} 
que existe cierta especialización de determinadas áreas del cerebro, parece que no hay un módulo determinado encargado de un tipo concreto de actividad sino que distintas áreas interactúan para su realización. El cerebro se entiende por ello como un sistema de redes neuronales que se van reorganizando continuamente. La comprensión y producción del lenguaje, al igual que otras funciones cerebrales, son procesos muy complejos que tienen lugar mediante la asociación y coordinación de información procedente de fuentes sensoriales distintas (vid. Heidemann, 1996).

Los conocimientos exactos sobre lo que ocurre en nuestro cerebro cuando aprendemos una lengua son, pues, todavía limitados. No obstante, se han desarrollado modelos de representación que intentan explicar cómo el cerebro analiza la información, cómo almacena y representa el conocimiento en la memoria o cómo lo activa y recupera. Así, por ejemplo, la psicología cognitiva distingue entre la memoria a corto plazo (o memoria de trabajo) y la memoria a largo plazo, entre memoria semántica y memoria episódica y entre conocimientos declarativos y conocimientos procedimentales (vid., por ejemplo, Glover, Ronning y Bruning, 1990). Sin embargo, el modelo que puede ser más esclarecedor a la hora de entender cómo adquirimos palabras nuevas cuando aprendemos una lengua es el del lexicón mental y, derivado de éste, el del lexicón mental multilingüe. Presentaremos ambos conceptos brevemente a continuación.

\subsubsection{El lexicón mental}

Por lexicón mental se entiende la estructura que permite el almacenamiento organizado de la información en la memoria a largo plazo. $^{2}$ Para entender su funcionamiento, se recurre frecuentemente a la analogía de una red muy compleja formada por nodos (los conceptos) interrelacionados por medio de conexiones múltiples. Cada concepto ${ }^{3}$ o categoría está asociado a información fonológica y grafemática, morfológica, sintáctica así como semántica (Börner y Vogel, 1994: 4). Las asociaciones semánticas se basan en diversos tipos de relaciones: en relaciones jerárquicas, en la frecuencia de coocurrencia, en la similitud del significado, etc. (Heidemann, 1996: 29). El conjunto de las relaciones de un concepto con otros conceptos constituye lo que entendemos por su significado. No se trata, pues, de un significado único e invariable: Las asociaciones suscitadas por una unidad léxica no son totalmente iguales para todos los hablantes de una lengua sino que son hasta cierto punto individuales de cada persona, puesto que las vamos configurando de acuerdo con nuestra experiencia lingüística y vital.

\section{Estructura de las categorías}

Estudios de psicología cognitiva (por ejemplo Rosch, 1973, 1975, 1978) han demostrado que las categorías de nivel básico -los conceptos más comunes- tienen una estructura prototípica, es decir que hay ejemplos más centrales y ejemplos más periféricos. Por ejemplo, la palabra «pájaro» hace pensar a la mayoría de hablantes del español en tipos de aves como el gorrión,

\footnotetext{
${ }^{2}$ Una introducción clásica y muy ilustrativa a este tema es la de Aitchison (2003).

${ }^{3}$ No distinguimos aquí entre concepto léxico o semántico (conocimiento lingüístico) y concepto cognitivo (conocimiento sobre el mundo). La controversia sobre la separación o no entre estos dos tipos de conocimientos no es relevante en el marco de este trabajo puesto que en el aprendizaje de una lengua extranjera es esencial la combinación de ambos.
} 
la paloma, la gaviota o similares - que serían elementos centrales de la categoría-, pero no tanto en un pingüino o un avestruz, los cuales, sin dejar de pertenecer a la categoría, ocupan una posición más periférica. Otros principios importantes de la categorización son: a) el hecho de que no suele haber bordes nítidos entre categorías sino que éstos son borrosos y b) que la relación entre miembros de una categoría se puede describir como una «semejanza de familia» en el sentido de Wittgenstein (1953). La semejanza de familia se refiere a que no tiene por qué haber un único rasgo semántico (o un grupo de ellos) que sea aplicable a todos los miembros de la categoría sino que la constelación de rasgos puede ser variable.

\section{Palabras y cultura}

Retomando el modelo del lexicón mental, podríamos resumir que, para un hablante concreto, el significado de una unidad léxica está formado por múltiples asociaciones y relaciones con otros conceptos, los cuales el hablante ha ido estableciendo y enriqueciendo a lo largo de su vida. Otro aspecto importante de esta visión experiencial del significado es que también incluye connotaciones, sensaciones, valoraciones, etc., y éstas pueden estar marcadas por la cultura. Por ejemplo, para un hablante nativo de alemán, la palabra Sonntag («domingo») no remite únicamente al concepto neutro «séptimo día de la semana» o «día de la semana entre sábado y lunes» sino a toda una serie de connotaciones específicas de la cultura alemana como Sonntagsfrühstück, Kirchenglocken, Familientag, Sonntagsspaziergang o Kaffee und Kuchen (el desayuno del domingo, las campanas, el día dedicado a la familia, el paseo del domingo o la merienda con café y tartas). Si el entorno en el que dos personas han crecido y se han formado lingüísticamente es similar, estos aspectos convencionales de los conceptos también serán similares. No obstante, si proceden de culturas diferentes, el significado connotativo que ambos relacionan con la misma palabra puede ser muy distinto e incluso llevar a malentendidos (pensemos, por ejemplo, en las palabras «nacionalista» - «nationalistisch»). De esta manera, palabras que son en apariencia totalmente equivalentes en dos lenguas pueden divergir bastante en los aspectos connotativos del significado (Wald / bosque, Brot / pan, August / agosto).

Por otra parte, la cultura también repercute sobre el aspecto mencionado en el punto anterior - la estructura de las categorías. Por ejemplo, si nos fijamos en el concepto «árbol», en la cultura alemana un roble o un castaño podrían ser considerados como árboles prototípicos, mientras que una palmera o un naranjo no serían ejemplos centrales de esta categoría en la lengua-cultura alemana. ${ }^{4}$

\subsubsection{El lexicón mental multilingüe: Similitudes y diferencias entre la adquisición léxica en la primera y en una segunda lengua}

Un campo de investigación relativamente reciente y muy interesante con vistas a la adquisición de segundas lenguas o lenguas extranjeras es el de la configuración del lexicón mental bilingüe o multilingüe (vid., por ejemplo, Schreuder y Weltens (1993), Singleton (1999)

\footnotetext{
${ }^{4}$ En el ámbito de la enseñanza del alemán como lengua extranjera, se han hecho estudios contrastivos interculturales sobre todo con referencia a culturas más lejanas, como la coreana. Así, Wollert (2002) muestra las diferencias en la estructura prototípica de algunos conceptos («leichtsinnig», «vorsichtig», «Reihenhaus», «altes Haus») en alemán y en coreano a través del uso que hacen sus estudiantes coreanos de estas palabras en alemán.
} 
o Wolter (2001). Algunas de las preguntas principales de estas investigaciones son, por ejemplo: la similitud o diferencia funcional o estructural entre el lexicón mental en la L1 y en la L2; así como la naturaleza y el grado de interconexión o integración del lexicón multilingüe, es decir, la cuestión de si consta de varios módulos o no y, en caso afirmativo, de qué forma están relacionados los diferentes componentes (vid. las aportaciones en Cenoz, Hufeisen y Jessner (2003)). Sin embargo, se trata de una cuestión que no es fácilmente accesible a la observación y, consecuentemente, los resultados parecen ser todavía demasiado provisionales (e incluso contradictorios) para sacar conclusiones para la enseñanza de lenguas.

En cambio, sí ha sido ampliamente estudiado el proceso de adquisición léxica en una segunda lengua, y aquí se pueden constatar algunas diferencias básicas en comparación con la adquisición de la primera lengua (Neuner-Anfindsen, 2005):

- El aprendiz de una segunda lengua se encuentra en una fase de evolución fisiológica y cognitiva más avanzada.

- El input que recibe un aprendiz de una segunda lengua es muy distinto al que recibe un niño que aprende su lengua materna.

- El aprendiz de una segunda lengua ya ha pasado por el aprendizaje de una lengua, por lo que ya ha superado las primeras fases de la evolución léxica.

Por otra parte, también hay puntos en común en la experiencia de aprendizaje de la L1 y la L2 (Singleton, 1999, citado en Neuner-Anfindsen, 2005). Por una parte, en lo que se refiere a la fonética, el aprendiz de una L2, igual que el aprendiz de L1, tiene que aprender a distinguir entre diferencias fonémicas y no fonémicas en la L2, tanto para la recepción como para la producción. Una dificultad añadida es que el aprendiz de la L2 ya dispone de un sistema fonológico de referencia (el de la L1), que puede ser causa de interferencias. Por otra parte, hay cierto paralelismo en el nivel semántico-conceptual: Aunque el aprendiz de L2 podrá recurrir en muchos casos a la estructura conceptual de la L1, también tendrá que aprender conceptos que son totalmente nuevos para él o que tienen sólo una correspondencia parcial con los conceptos ya existentes de la L1.

\subsection{Las palabras en su contexto}

Otro aspecto de las palabras que está empezando a ser tenido en cuenta en las aplicaciones didácticas es la interrelación entre la unidad léxica y el contexto, es decir, el hecho de que en el lenguaje las palabras no aparecen de forma aislada sino siempre en compañía de otras y que las posibilidades de combinación no son aleatorias. La interrelación entre el significado de la unidad léxica y el contexto se manifiesta de dos maneras diferentes y complementarias. Para apreciar esta doble relación, conviene distinguir entre el significado léxico de una palabra y el significado textual. El primero abarca todas las posibilidades de significado de la unidad léxica en cuestión. Cuando se utiliza la unidad léxica en un contexto o una situación dada, en cambio, entre todos estos significados potenciales se actualizará uno muy concreto (y más restringido en la mayoría de los casos): el significado textual (vid., por ejemplo, Matussek, 1994). Este es el primer tipo de relación (ilustración 1): En el nivel individual de la aparición de una unidad léxica en un contexto concreto solamente se activará la porción relevante del significado léxico (es decir, el significado textual). 
Ilustración 1: Actualización del significado por influencia del contexto

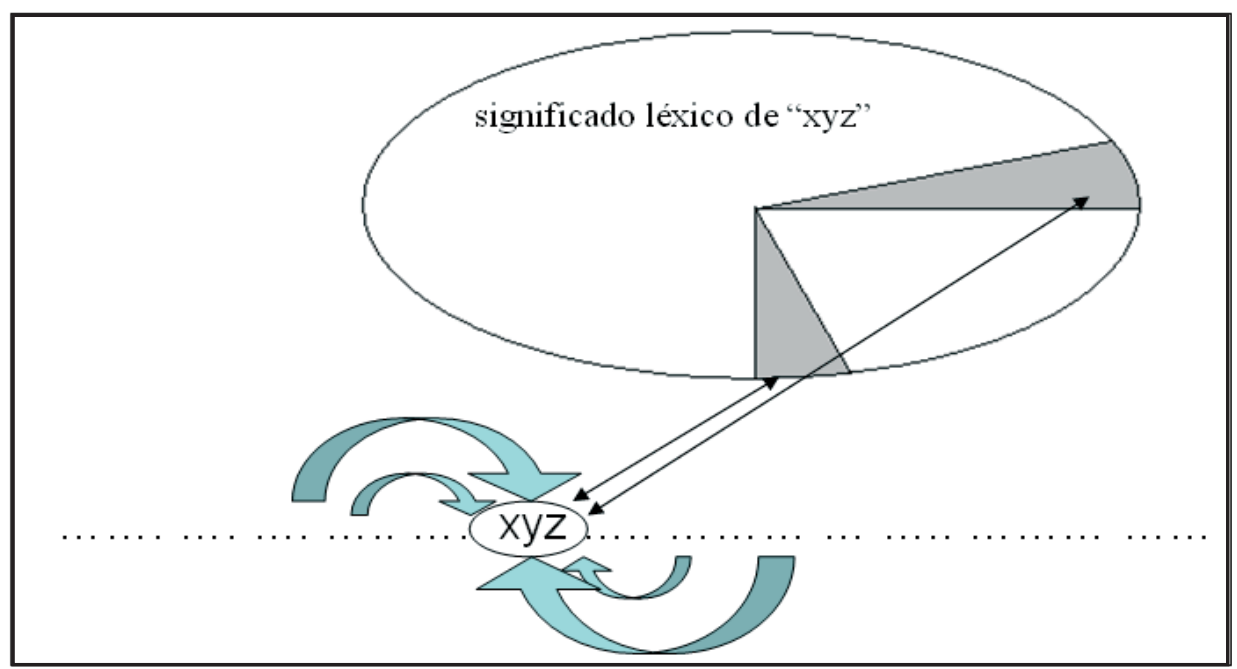

Por otra parte, en el nivel general del significado léxico, la suma de los contextos -y con ello nos adentramos de lleno en el nivel del uso- repercute en el significado de la unidad léxica (ilustración 2).

Ilustración 2: Influencia del uso de las unidades léxicas sobre su significado

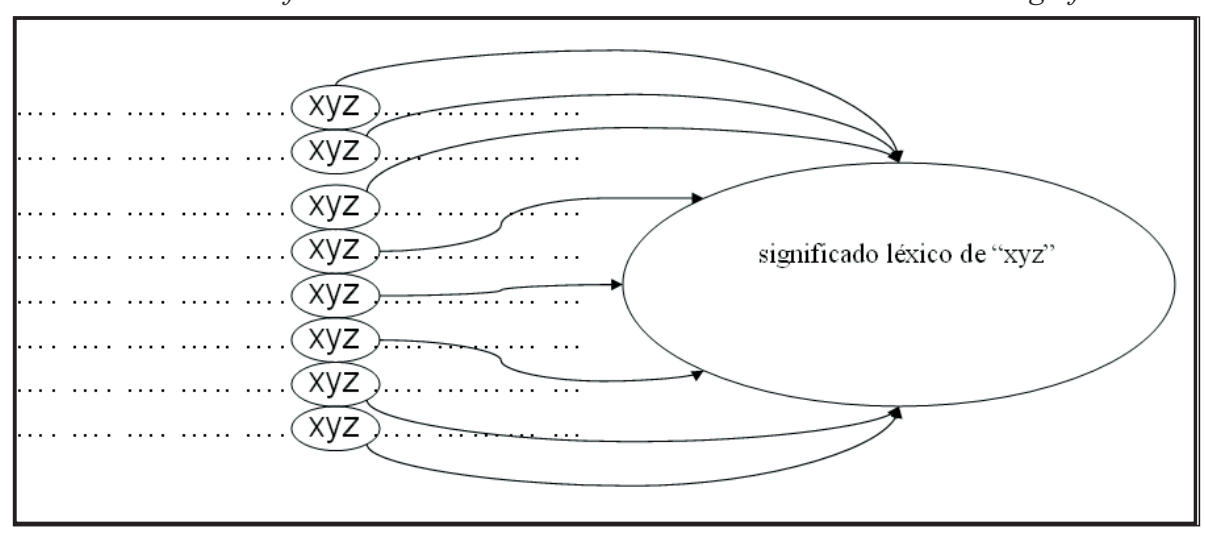

Fueron Sinclair y sus colaboradores, inspirados en el contextualismo británico inaugurado por Firth $(1957,1969)$ y apoyándose en la metodología del análisis de corpus, quienes proporcionaron amplias muestras de la gran importancia de las coocurrencias de unidades léxicas para el significado de éstas. En primer lugar, Sinclair (1991) propone el «principio idiomático» (idiom principle) en vez del «principio de la libre elección» (open-choice principle), según el cual la única limitación a la combinación de palabras sería la gramaticalidad. De acuerdo con el principio idiomático, los hablantes tenderán a utilizar determinadas combinaciones de 
palabras con una frecuencia mucho mayor que otras, de manera que surge una gran cantidad de combinaciones léxicas «semiprefabricadas» que funcionan como unidades en el lenguaje, lo cual lleva a una fijación de las combinaciones no solamente en el uso sino también en el lexicón mental de los hablantes.

Sinclair (1996) llama estas combinaciones de unidades léxicas «unidades extendidas de significado» (extended units of meaning) y describe los siguientes aspectos del significado relacionados con las coocurrencias:

- el perfil colocacional (la capacidad de combinación léxica)

- los patrones coligacionales (las combinaciones lexicogramaticales)

- la preferencia semántica (la combinación con unidades de determinados campos semánticos)

- la prosodia semántica (el aspecto pragmático de valoración positiva o negativa implícita)

Cada una de estas vertientes de las «unidades extendidas de significado» limita la capacidad de combinación de estas con otras palabras en un sentido concreto y estrecha sus lazos con determinados grupos de palabras. De esta manera, el aspecto colocacional llega a formar parte del significado de una unidad. Entre los cuatro aspectos mencionados, es evidente la utilidad de las colocaciones para el aprendiz de una lengua extranjera ya que el uso adecuado de las mismas le permite expresarse con mayor naturalidad. Sin embargo, también nos parece especialmente interesante la contribución de las coocurrencias al significado de una palabra, ya sea por las preferencias semánticas de la unidad o al conferir o recibir implícitamente una valoración positiva o negativa (prosodia semántica). ${ }^{5}$

\section{REPERCUSIONES SOBRE EL PROCESO DE ENSEÑANZA-APRENDIZAJE}

Es bien sabido que la adquisición de vocabulario es un proceso lento que requiere repetición, reorganización de la información así como una combinación de distintos tipos y situaciones de aprendizaje. Nation (2001) lo formula así:

Learning a word is a cumulative process. We cannot expect a word to be learned in one meeting and so we need to see each meeting as a small contribution to learning. Learning a word occurs across a range of different learning conditions, $[\ldots]$ those conditions should involve roughly equal proportions of the four strands of meaning-focused input, language-focused input, meaning-focused output and fluency development. (Nation, 2001: 296)

Por ello, se recomienda en general utilizar una combinación de ejercicios orientados, por una parte, a la fluidez (actividades con un fin comunicativo, para facilitar el acceso mental a la palabra) y, por la otra, a la riqueza, con el fin de incrementar la cantidad y variedad de asociaciones relacionadas con ella (Nation y Newton, 1997). Basándonos en los dos bloques

\footnotetext{
${ }^{5}$ Algunos estudios pioneros que ilustran el efecto de prosodia semántica son Sinclair (1987), Louw (1993) o Stubbs (1995).
} 
expuestos en el apartado anterior -el almacenamiento de los conceptos léxicos en el cerebro y el comportamiento de las palabras en contexto- mostraremos ahora de qué manera estos diferentes aspectos han sido aplicados a la adquisición y la enseñanza de lenguas extranjeras y cómo están relacionados con la fluidez y con la riqueza del conocimiento léxico.

\subsection{Estrategias de aprendizaje de vocabulario basadas en el funcionamiento del lexicón mental}

Aunque queden muchos detalles por resolver en lo que se refiere a la representación mental de la información lingüística tanto en un individuo monolingüe como en uno bilingüe, hay un dato básico que tiene repercusiones muy claras sobre el aprendizaje de una lengua extranjera: el hecho de que la organización de los conceptos en nuestro cerebro se basa en la interconexión y de que el significado de los elementos se define por sus relaciones múltiples con otros conceptos. De ello se desprende que cuanto más relacionado esté un concepto con otros conceptos de diversa índole, más fácilmente podrá ser activado. Para la adquisición de vocabulario en una lengua extranjera, esto significa que la retención y el acceso a las palabras serán mejores si durante el aprendizaje:

- se establecen múltiples conexiones con otras palabras,

- se estructura la información (estableciendo distintos tipos de relaciones),

- se involucran varios sentidos (ver, oír, etc.) o formatos de presentación de la información (texto, imágenes).

En este último punto debemos tener en cuenta que no todos los aprendices emplean con igual eficiencia los diferentes sentidos. Se han descrito distintos tipos de aprendices dependiendo del canal sensorial que les resulta más importante para el aprendizaje. De esta forma se ha distinguido entre los tipos visual, auditivo, háptico y olfativo. Sin duda, se trata de una simplificación y en la realidad todos empleamos una combinación de varios estilos (con cierta preferencia por alguno). No obstante, es un argumento más para intentar proporcionar al estudiante información que apele a varios de sus sentidos y, además, no solamente a sus capacidades cognitivas sino también a su capacidad emocional, puesto que se ha demostrado que las emociones son un activador potente de la memoria.

\subsection{Analizar la «carga» cultural de las palabras}

También el aspecto de la especificidad cultural de la categorización (vid. 2.1.1) se está teniendo en cuenta en la enseñanza de lenguas extranjeras. Una de las propuestas más elaboradas en este sentido es la de Müller (1994), quien argumenta que, con el fin de evitar malentendidos interculturales debidos a la transferencia de connotaciones de la cultura propia a palabras de la lengua y cultura extranjeras, se debería sensibilizar sistemáticamente a los aprendices de una lengua extranjera respecto a la existencia de estas diferencias. ${ }^{6}$ Propone

\footnotetext{
${ }^{6}$ Para ello analiza una serie de conceptos que pueden llevar una carga convencional cultural considerable: Zeitung (periódico), Familie (familia), Beruf (profesión), Haus (casa), gerecht (justo), logisch (lógico), Kommunismus (comunismo), etc.).
} 
para este fin una metodología para familiarizarse con los aspectos convencionales de los conceptos, basada en un catálogo de preguntas que el aprendiz se va haciendo a sí mismo para detectar posibles rasgos específicos.

Una forma original de profundizar en aspectos culturales del vocabulario es la propuesta de Shaughnessy y Parkhill $(2003)^{7}$ de un diccionario pictórico «culturalmente auténtico». Se trata de una página web que permite trabajar el vocabulario (alemán, en este caso) con la ayuda de imágenes, con la peculiaridad de que las fotografías son "culturalmente auténticas», es decir, se muestran ejemplos prototípicos en la cultura alemana de un árbol, pan, ropa, etc. ${ }^{8}$ De esta manera se combina el aspecto de la activación de canales diferentes (imágenes y texto) con el de la influencia de la cultura en la configuración de las categorías.

\subsection{El aprendizaje del vocabulario en contexto: Trabajo con colocaciones y concordancias}

Un número creciente de autores (por ejemplo Lewis, 1993, 1997; Nation y Newton, 1997; Hoey, 2000 a y b; Schmitt, 2000; Nation, 2001) tienen en cuenta el aspecto de colocación (o el de coocurrencia) y proponen ejercicios de vocabulario cuya finalidad es la de incrementar la conciencia de los aprendices respecto a la probabilidad de que las palabras aparezcan en compañía de determinadas otras palabras. Nation (2001: 336-342), por ejemplo, sugiere el fomento del chunking (la conciencia y el uso de colocaciones en el sentido más amplio de la palabra). También en este contexto encontramos la distinción entre tareas encaminadas a incrementar la fluidez por una parte y a la riqueza por otra. Las primeras incluyen actividades de lectura rápida y extensiva así como ejercicios orales que obligan a la memorización de segmentos, como leer frases levantando la vista del papel, leer una frase para copiarla sin volver a mirar, etc. El objetivo de las actividades del segundo tipo, en cambio, es sobre todo el de orientar la atención de los aprendices hacia el funcionamiento de la lengua a través del análisis de concordancias (data-driven learning, vid., sobre todo, Johns y King, 1991; Johns, 2002). Desde el punto de vista metodológico, en la utilización de corpus electrónicos para la enseñanza de lenguas extranjeras encontramos enfoques muy diferentes que van desde la utilización de datos extraídos de corpus que permiten un aprendizaje inductivo, pero controlado y guiado por el profesor (como las propuestas de Johns, 1994, por ejemplo) hasta actividades orientadas a fomentar la autonomía. Entre estas últimas podemos encontrar la búsqueda libre de información lingüística (Bernardini, 2000; 2001; 2002; 2004).

A pesar de que se ha mostrado que el análisis de concordancias por parte de los estudiantes es efectivo para el aprendizaje de vocabulario (Cobb, 1997), en las aplicaciones didácticas suelen primar los aspectos gramaticales. Ello se debe probablemente a que es relativamente fácil deducir una regularidad gramatical a partir de un número reducido de ejemplos. En cambio, no abundan las propuestas relacionadas con el vocabulario, posiblemente porque es mucho más difícil extraer información semántica de un listado de concordancias. Por este motivo queremos proponer a continuación una actividad que ilustra el uso de corpus como herramienta para la adquisición de vocabulario.

\footnotetext{
${ }^{7}$ La propuesta está accesible en: http://www.washjeff.edu/capl/.

${ }^{8}$ Vid. Macaire y Hosch (1996) sobre el papel de las imágenes en la adquisición de conocimientos culturales.
} 


\section{Ejemplo de aCTIVIDAd didÁCTICA}

La conclusión principal que podríamos extraer de la exposición anterior es que los diferentes enfoques comparten la idea central de que una característica fundamental de las unidades léxicas es su interrelación con otras unidades. La importancia de estas relaciones radica, por una parte, en que de ellas depende su significado y, por otra, que resultan tan fuertes que determinan en gran medida su uso. Por ello la actividad didáctica que presentaremos no comparte únicamente las características del data-driven learning de seguir un procedimiento inductivo y perseguir el aprendizaje por descubrimiento sino que intenta, además, retomar algunas de las ideas citadas más arriba sobre aspectos clave de la adquisición de vocabulario: la especificidad cultural de las palabras, la relación entre significado y contexto, así como la importancia que tienen para el significado las relaciones estructuradas que unas unidades léxicas mantienen con otras. Para ello recurrimos a dos conceptos de la lingüística de corpus (vid. 3.3.2) que resultan muy útiles para aproximarnos al significado de una palabra a través del análisis de sus coocurrencias: la prosodia y la preferencia semántica. ${ }^{9}$

Un elemento imprescindible para poder aprovechar estos conceptos en la enseñanza de lenguas extranjeras es la existencia de grandes corpus electrónicos fácilmente accesibles y que ofrezcan una interfaz de consulta fácil de usar y que ofrezca la información relevante sin necesidad de formular búsquedas complejas. Afortunadamente, cada vez disponemos de más recursos de este tipo. Aun así, la utilización didáctica de corpus electrónicos no resulta fácil en grupos de estudiantes con niveles de competencia media o baja en la lengua extranjera. Por ello es necesaria una introducción gradual de los corpus en la clase de lengua extranjera, con una adquisición paulatina de las competencias necesarias para su manejo (vid. Oster, 2007). En una primera fase, aunque se persigue el fomento de un aprendizaje inductivo a través del análisis de material auténtico, la intervención del profesor, que selecciona los contextos y guía la actividad, deberá ser todavía considerable. En una fase más avanzada, de mayor autonomía del estudiante, éste puede tener acceso libre a corpus electrónicos y realizar actividades pautadas por el profesor.

La actividad que proponemos a continuación abarca ambas fases, guiando a los estudiantes en la realización de un análisis semántico contrastivo de unidades léxicas aparentemente equivalentes (falsos amigos). La actividad está diseñada para estudiantes con un nivel B1 de alemán, aproximadamente. Tiene formato de webquest (o cibertarea) ${ }^{10} \mathrm{y}$ fue realizada en una prueba piloto por un grupo de estudiantes de la Universitat Jaume I de Castellón. Con el fin de poder valorar el funcionamiento de la actividad, los estudiantes realizaron una evaluación de la misma que comentaremos en el apartado 4.2.

\subsection{Descripción de la actividad: Los falsos amigos en los diccionarios bilingües y en corpus}

La actividad contribuye principalmente a los siguientes objetivos:

- Iniciarse en el uso de corpus electrónicos: familiarización con conceptos básicos (coocurrencias, vecinos derechos e izquierdos, etc.)

\footnotetext{
${ }^{9}$ Vid. Oster y van Lawick (en prensa) para una propuesta metodológica sobre la utilización de estos conceptos para el análisis semántico en clase.

${ }^{10}$ Se puede acceder a ella en esta dirección: http://www3.uji.es/ oster/plantilla2/index.htm.
} 
- Reconocer el papel que desempeñan las coocurrencias para la configuración del significado de las unidades léxicas

- Buscar de forma activa los puntos en común y el contraste de las lenguas de trabajo

Para poder realizar la actividad, se requiere un aula con ordenadores con acceso a Internet así como diccionarios bilingües (en papel y/o electrónicos).

El corpus elegido para el análisis de las palabras alemanas (Wortschatz) ${ }^{11}$ ofrece la ventaja de que es en realidad una combinación de corpus y diccionario monolingüe y que, aparte de una cantidad muy grande de ejemplos en contexto, ofrece un análisis ya hecho de las coocurrencias más frecuentes. Por este motivo, esta herramienta se puede emplear para actividades sencillas incluso con aprendices que no tienen un nivel muy alto de alemán, puesto que pueden acceder directamente a las listas de los «vecinos» derechos e izquierdos más frecuentes sin necesidad de hacer análisis complejos. En el caso de $\mathrm{CREA}^{12}$, el corpus de referencia del español actual de la Real Academia, utilizado para el análisis en español, las búsquedas son más laboriosas puesto que el corpus no ofrece la misma facilidad de uso ni de análisis de coocurrencias ya realizados. No obstante, al tratarse de la lengua materna de los estudiantes no les resulta demasiado complicado interpretar las concordancias.

\section{Primera fase: Introducción}

Debate inicial de la problemática de los falsos amigos. Los estudiantes nombran ejemplos de falsos amigos. Por medio de los ejemplos se realiza una primera distinción entre los siguientes tipos:

- aquellos que tienen un significado totalmente diferente (Ejemplo: alemán Frieden inglés freedom - paz - libertad)

- aquellos cuyos significados tienen cierto parecido (Ejemplo: alemán sensibel - inglés - sensible sensible - razonable)

\section{Segunda fase: Clasificación de falsos amigos}

Los estudiantes trabajan la ficha 1 en pequeños grupos. ${ }^{13}$

En primer lugar, los estudiantes analizan las palabras en negrita de las frases de ejemplo. Para ello identifican el posible falso amigo en español y buscan la palabra en el diccionario bilingüe. Con ayuda del contexto deciden si el falso amigo expresa algo similar o algo totalmente distinto y si la propuesta del diccionario es adecuada o si habría que buscar una equivalencia mejor para el contexto. Cabe destacar aquí que en los tres casos de similitud de significado (Karaffe - garrafa, diskutieren - discutir, ordinär - ordinario), algunos diccionarios $^{14}$ ofrecen el falso amigo como equivalencia. Aunque es indudable que existe cierto solapamiento de significado, esta información del diccionario bilingüe dista de ser óptima para facilitar la comprensión de estas unidades léxicas.

\footnotetext{
${ }^{11}$ El corpus Wortschatz, compilado por la Universidad de Leipzig, contiene 500 millones de palabras y es accesible libre y gratuitamente a través de Internet (http://wortschatz.uni-leipzig.de).

${ }^{12}$ Accesible en http://corpus.rae.es/creanet.html.

${ }^{13}$ Para facilitar la comprensión, hemos añadido entre corchetes una traducción de las instrucciones en cursiva, así como las soluciones aportadas con mayor frecuencia por los estudiantes.

${ }^{14}$ Reflejamos aquí las propuestas de Langenscheidts Handwörterbuch Spanisch (1998).
} 


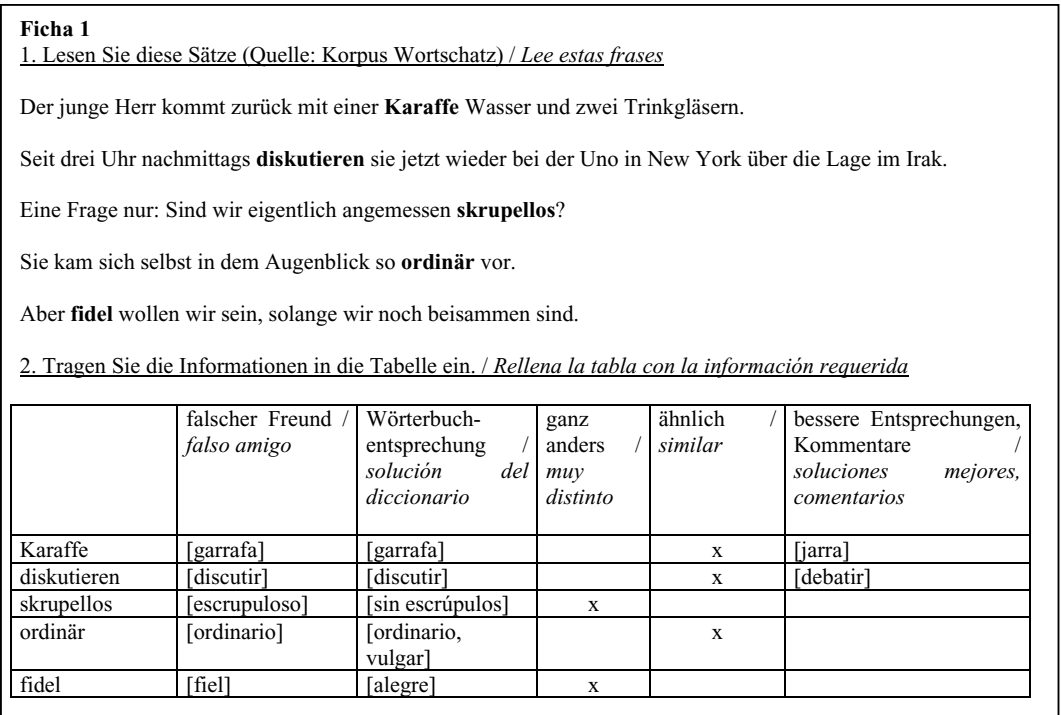

Tercera fase: Análisis de Karaffe

Por ello, en este momento se recurre a una obra de consulta distinta (el corpus electrónico), con el fin de averiguar si esta herramienta permite obtener información semántica más precisa sobre la palabra Karaffe y las equivalencias de «garrafa» o «jarra». Primero, los estudiantes realizan un análisis de la palabra Karaffe en el corpus alemán Wortschatz con ayuda de la ficha 2. El corpus en cuestión proporciona cuatro tipos de información: una descripción, palabras relacionadas, colocaciones y contextos. De esta forma, se puede averiguar lo siguiente ( vid. las soluciones en la ficha 2):

a) Karaffe es una «botella de cuerpo ancho», «botella de cristal tallado».

b) La palabra es sinónima de otras que significan contenedor, botella, recipiente, jarra.

c) Las colocaciones, en particular los «vecinos derechos», ofrecen información sobre tipos de contenido así como materiales frecuentes de este tipo de recipiente. En este caso, los contenidos más habituales son vino, chianti, agua y vino tinto y los materiales cristal y plata. ${ }^{15}$ Finalmente, revisando algunos contextos, se llega a la conclusión de que se trata de un recipiente valioso o de contenido valioso, por ejemplo un buen vino y se encuentran pistas de que una función de este tipo de recipiente es la de decantar el vino.

\section{Cuarta fase: Análisis de garrafa y jarra}

Con esta información sobre la palabra alemana Karaffe, se pasa a hacer el mismo análisis con los posibles equivalentes españoles garrafa y jarra (ficha 3). Habiendo analizado tanto la definición que ofrece el diccionario monolingüe de la RAE como las concordancias y las agrupaciones de palabras que ofrece el corpus CREA, y comparando estos datos con los del análisis de Karaffe, se llega a la conclusión siguiente (vid. las soluciones en la ficha 3):

\footnotetext{
${ }^{15}$ Al tratarse de un corpus en continuo crecimiento, los resultados de las búsquedas pueden variar a lo largo del tiempo, aunque no deberían hacerlo sustancialmente.
} 
- En el caso de garrafa no coincide ni la forma (cuello y boca anchos, con asas) ni el tamaño (voluminoso) ni la calidad del contenido (bebida alcohólica de mala calidad, gasolina, agua, gas, vino). ${ }^{16}$

- En el caso de jarra coinciden la forma (cuello y boca anchos), el material (cristal) y el contenido al menos en parte (agua, cerveza, vino, limonada) aunque jarra no parece conllevar las mismas connotaciones de calidad que el alemán Karaffe.

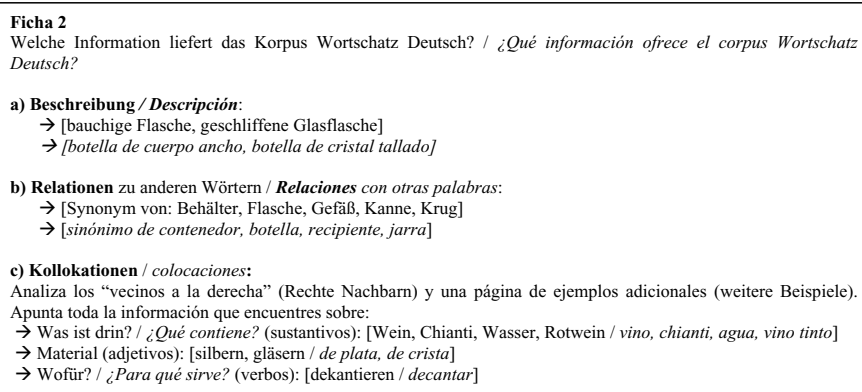

\begin{tabular}{|c|c|c|c|}
\hline & jarra & garrafa & Karaffe \\
\hline Contenido & $\begin{array}{l}\text { [agua, cerveza, vino, } \\
\text { café, leche] }\end{array}$ & $\begin{array}{l}\text { [agua, vino, aceite, } \\
\text { gasolina, gas, anís, licor] }\end{array}$ & $\begin{array}{l}\text { [vino, chianti, agua, vino } \\
\text { tinto] }\end{array}$ \\
\hline Material & $\begin{array}{l}\text { [cristal, cobre, porcelana, } \\
\text { barro] }\end{array}$ & -- & $\begin{array}{l}\text { [cristal tallado, cristal, } \\
\text { plata] }\end{array}$ \\
\hline Tamaño & -- & [de cinco litros] & -- \\
\hline Forma & $\begin{array}{l}\text { [cuello y boca anchos, } \\
\text { una o dos asas] }\end{array}$ & $\begin{array}{l}\text { [esférico, cuello largo y } \\
\text { estrecho] }\end{array}$ & [de cuerpo ancho] \\
\hline $\begin{array}{l}\text { Calidad del contenido } \\
\text { o contenedor }\end{array}$ & -- & [mala, a granel] & [valioso] \\
\hline Utilidad & [servir bebidas] & $\begin{array}{l}\text { [transportar líquidos, } \\
\text { enfriar bebidas] }\end{array}$ & $\begin{array}{l}\text { [servir bebidas, escanciar } \\
\text { vino] }\end{array}$ \\
\hline
\end{tabular}

Conclusión:

¿Cuál de las dos palabras es más parecida a Karaffe en cuanto al significado? ¿Cuáles son las diferencias y las similitudes?

\footnotetext{
${ }^{16}$ Como su propio nombre indica, el CREA refleja el español actual. Una búsqueda en un corpus que permita restringir la búsqueda a textos más antiguos (como el Corpus del Español: http://www.corpusdelespanol.org) podría dar resultados ligeramente diferentes.

${ }^{17}$ Curiosamente, aquellos que llegaron a la conclusión equivocada sí que realizaron el análisis de forma correcta y dos de ellos calificaron el trabajo con el corpus alemán como «perfectamente factible» (vid. item 4).
} 


\subsection{Resultados y evaluación de la experiencia en clase}

La actividad se ha realizado como una experiencia piloto con un grupo de 31 estudiantes de la licenciatura de Traducción e Interpretación (Alemán Lengua C), de un nivel de alemán aproximado de B1. Al ser uno de los objetivos principales de la actividad el guiar a los estudiantes hacia un uso autónomo de las herramientas, la tarea se presentó en formato webquest (o «cibertarea»), que permite que cada estudiante realice la actividad a su ritmo y con todos los recursos necesarios. Dada esta situación de clase, la intervención de la profesora fue mínima, no obstante lo cual los estudiantes pudieron seguir adecuadamente todos los pasos y la gran mayoría (27 de 31) llegó al resultado deseado (la diferenciación semántica entre los falsos amigos Karaffe/garrafa). ${ }^{17}$ De esta manera, lograron familiarizarse de un modo práctico con aspectos importantes del significado que solamente se aprecian a través de las coocurrencias de las unidades léxicas, como la preferencia semántica (en este caso: los diferentes tipos de contenido de garrafa/jarra/Karaffe) y la prosodia semántica (aquí: la valoración positiva de contenido y contenedor en el caso de Karaffe).

Finalmente, a través de un cuestionario, los estudiantes valoraron algunos aspectos de la tarea con los siguientes resultados (vid. ilustración 3):

- Más de la mitad afirmaron que habían aprendido mucho sobre los corpus, y otro $36,7 \%$ que habían aprendido bastante.

- Todos calificaron la información proporcionada por los corpus como relevante, valorándola la mitad de ellos como más o mucho más relevante que la de los diccionarios bilingües, y la otra mitad como igual de relevante.

- Casi todos consiguieron trabajar con el corpus CREA sin mayores problemas (un $95 \%$ lo ve «factible»).

- Como es de esperar, trabajar con un corpus monolingüe alemán resulta más difícil, pero también aquí un $61 \%$ lo ve «difícil pero factible» y un $12 \%$ «perfectamente factible».

Como ya hemos resaltado, aunque a primera vista esta actividad trata del tema de los falsos amigos, éste no es su objetivo principal. Lo que pretendemos sobre todo es una primera aproximación a un tipo de obra de consulta complejo y al que los estudiantes posiblemente no recurrirían por la dificultad que entraña su utilización. En este contexto, nos parece especialmente alentadora la buena acogida y valoración del trabajo con corpus electrónicos por parte de los participantes en la experiencia. 
Ilustración 3: Valoración del trabajo con corpus por parte de los estudiantes

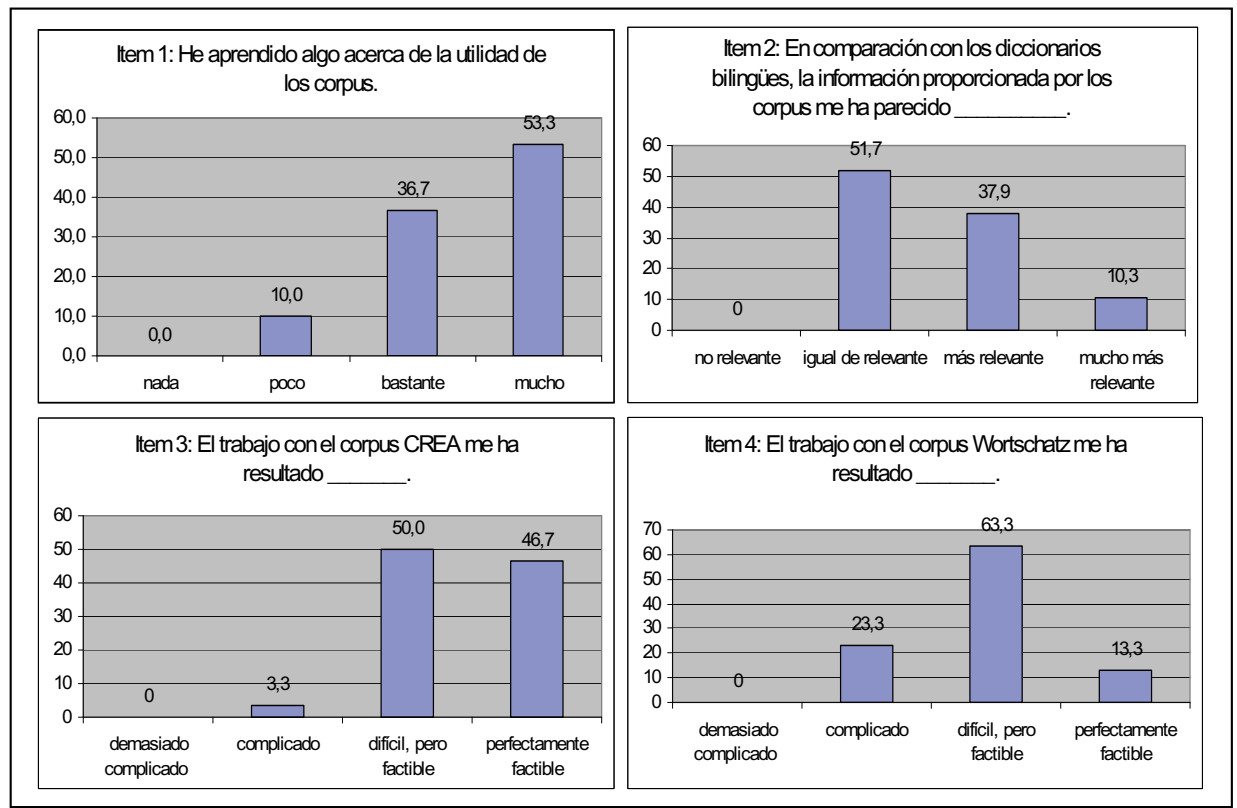

\section{Conclusiones}

En este artículo nos planteábamos revisar algunas de las aportaciones teóricas más relevantes para el desarrollo de la competencia léxica en una lengua extranjera. En estas investigaciones de diversos campos se aprecia un denominador común que consiste en la importancia concedida a la interrelación entre las unidades léxicas y la gran relevancia que estas relaciones tienen para el significado: Por una parte, el modelo del lexicón mental de la psicología cognitiva entiende el significado de una unidad léxica como el conjunto de las múltiples asociaciones y relaciones que esta mantiene con otros conceptos; y por otra parte, visto desde la perspectiva del uso, la lingüística de corpus nos ha mostrado que las unidades léxicas prefieren la compañía de unas palabras sobre la de otras, lo cual se expresa en la preferencia por determinadas colocaciones y en restricciones de uso. Y es más, la coocurrencia frecuente con determinadas otras unidades puede producir una influencia recíproca sobre los respectivos significados. De ahí se deriva la importancia de tratar la adquisición del vocabulario en la clase de lengua extranjera como la familiarización con entes «sociables» y no con unidades aisladas. Desarrollar la competencia léxica implica explorar cómo estos entes se relacionan, cuáles se llevan a la perfección, entre cuáles hay roces o saltan chispas (que generan significados inesperados) y cuáles son incompatibles; y entender que para comprender una palabra es útil el viejo dicho de «dime con quién andas y te diré quién eres». Para aprender a apreciar estas características de las palabras resulta muy útil y revelador el trabajo con corpus electrónicos. No obstante, la utilización de este tipo de herramientas en clase no es sencilla, por lo que creemos necesario el desarrollo de actividades que logren familiarizar paulatinamente a los 
estudiantes con algunos conceptos y métodos necesarios para el trabajo con corpus como recurso léxico.

\section{Bibliografía}

Aitchison, J. (2003). Words in the Mind: An Introduction to the Mental Lexicon (3 ${ }^{a}$ edición). Oxford: Basil Blackwell.

Bernardini, S. (2000). Competence, Capacity, Corpora: a Study in Corpus-aided Language Learning. Bologna: CLUEB.

Bernardini, S. (2000). «Systematising serendipity: Proposals for concordancing large corpora with language learners», in Burnard, L. and T. McEnery (eds.), Rethinking language pedagogy from a corpus perspective. Papers from the Third International Conference on Teaching and Language Corpora. Frankfurt a. M.: Peter Lang, 225-234.

Bernardini, S. (2001). «'Spoilt for choice’: a learner explores general language corpora», in Partington, A. (ed.). Learning with corpora. Houston: Athelstan, 220-249.

Bernardini, S. (2002). «Exploring New Directions for Discovery Learning», in Kettemann, B. y G. Marko (eds.), Teaching and Learning by Doing Corpus Analysis. Amsterdam/New York: Rodopi, 165-182.

Bernardini, S. (2004). «Corpora in the classroom», in Sinclair, J. (ed.). How to use corpora in language teaching. Amsterdam: Benjamins, 15-36.

Börner, W. y K. Vogel (1994). «Mentales Lexikon und Lernersprache», in Börner, W. and K. Vogel (eds.), Kognitive Linguistik und Fremdsprachenerwerb. Das mentale Lexikon. Tübingen: Gunter Narr.

Cenoz, J., B. Hufeisen and U. Jessner (eds.) (2003). The Multilingual Lexicon. Dordrecht: Kluwer Academic Publishers.

Coady, J. et al. (1993). «High Frequency Vocabulary and Reading Proficiency in ESL Readers», in Huckin, T., M. Haynes and J. Coady (eds.), Second language reading and vocabulary learning. Norwood, New Jersey: Ablex, 217-228.

Cobb, T. (1997). «Is there any measurable learning from hands-on concordancing?» in System, 25, 3: $301-315$.

Firth, J.R. (1957/1968). «A synopsis of linguistic theory, 1930-55», in Palmer, F.R. (ed.). Selected Papers of John Firth 1952-59. London: Longmans, 168-205.

Firth, J.R. (1969). Papers in Linguistics 1934-1951. London: Oxford University Press.

Glover, J.A., R.R. Ronning y R.H. Bruning (1990). Cognitive Psychology for Teachers. New York: Macmillan.

Haynes, M. (1993). «Patterns and Perils of Guessing in Second Language Reading», in Huckin, T., M. Haynes and J. Coady (eds.), Second Language Reading and Vocabulary Learning. Norwood, New Jersey: Ablex, 46-66.

Heidemann, A. (1996). The visualization of foreign language vocabulary in CALL. Frankfurt a. M.: Peter Lang.

Hoey, M. (2000). «The hidden lexical clues of textual organisation: a preliminary investigation into an unusual text from a corpus perspective», in Burnard, L. and T. McEnery (eds.), Rethinking Language Pedagogy from a Corpus Perspective. Frankfurt a. M.: Peter Lang, 31-41.

Hoey, M. (2000). «A world beyond collocation: new perspectives on vocabulary teaching», in Lewis, M. (ed.). Teaching Collocation. Further Developments in the Lexical Approach. Hove: LTP, 224-243. 
Johns, T. (1994). «From Printout to Handout: Grammar and Vocabulary Teaching in the Context of Data-driven Learning», in Odlin, T. (ed.). Perspectives on Pedagogic Grammar. Cambridge: CUP, 293-313.

Johns, T. (2002). «Data-driven Learning: The Perpetual Challenge», in Kettemann, B. and G. Marko (eds.), Teaching and Learning by Doing Corpus Analysis. Amsterdam/New York: Rodopi, 107-117.

Johns, T. and P. King (eds.) (1991). Classroom Concordancing. (English Language Research Journal 4). Birmingham: Birmingham University.

Lewis, M. (1993). The lexical approach. Hove: LTP.

Lewis, M. (1997). Implementing the lexical approach. Hove: LTP.

Louw, M. (1993). «Irony in the Text or Insincerity in the Writer? The Diagnostic Potential of Semantic Prosodies», in Baker, M., G. Francis and E. Tognini-Bonelli (eds.), Text and Technology: In Honour of John Sinclair. Amsterdam, Philadelphia: Benjamins, 240-251.

Macaire, D. and W. Hosch (1996). Bilder in der Landeskunde. Berlin: Langenscheidt.

Müller, B.-D. (1994). Wortschatzarbeit und Bedeutungsvermittlung. Berlin: Langenscheidt.

Nation, I.S.P. (2001). Learning vocabulary in another language. Cambridge: Cambridge University Press.

Nation, P. (2005). «Teaching Vocabulary», in Asian EFL Journal, 7, 3: 47-54.

Nation, P. and J. Newton (1997). «Teaching vocabulary», in Coady, J. and T. Huckin (eds.), Second Language Vocabulary Acquisition. Cambridge: Cambridge University Press, 238-255.

Neuner-Anfindsen, S. (2005). Fremdsprachenlernen und Lernerautonomie. Sprachlernbewusstsein, Lernprozessorganisation und Lernstrategien zum Wortschatzlernen in Deutsch als Fremdsprache. Baltmannsweiler: Schneider Verlag Hohengehren.

Oster, U. (2007). «Working towards autonomy: Corpora in the translation classroom», in Wotjak, G. (ed.). Quo vadis Translatologie? Ein halbes Jahrhundert universitäre Ausbildung von Dolmetschern und Übersetzern in Leipzig. Rückschau, Zwischenbilanz und Perspektiven aus der Außensicht. Berlin: Timme, 311-325.

Oster, U. and H. van Lawick (en prensa). «Semantic preference and semantic prosody: A corpusbased analysis of translation-relevant aspects of the meaning of phraseological units», in Thelen, M. and B. Lewandowska-Tomaszczyk (eds.), Translation and Meaning. Part 8. Maastricht: Hogeschool Zuyd, Maastricht School of Translation and Interpreting.

Rosch, E. (1973). «On the Internal Structure of Perceptual and Semantic Categories», in Moore, T.E. (ed.). Cognitive Development and Acquisition of Language. New York: Academic Press.

Rosch, E. (1975). «Cognitive Representations of Semantic Categories», in Journal of Experimental Psychology, 104: 192-233.

Rosch, E. (1978). «Principles of Categorization», in Rosch, E. and B.B. Lloyd (eds.), Cognition and Categorization. Hillsdale, N.J.: Erlbaum, 27-47.

Schmitt, N. (2000). Vocabulary in Language Teaching. Cambridge: Cambridge University Press.

Schreuder, R. and B. Weltens (eds.) (1993). The Bilingual Lexicon. Amsterdam / Philadelphia: John Benjamins.

Seidlhofer, B. (2002). «Pedagogy and local learner corpora», in Granger, S., J. Hung and S. PetchTyson (eds.), Computer Learner Corpora, Second Language Acquisition and Foreign Language Teaching. Amsterdam/Philadelphia: John Benjamins, 213-234.

Sinclair, J. (1987). «Collocation: a progress report», in Steele, R. and T. Threadgold (eds.), Language Topics. Essays in Honour of Michael Halliday. Amsterdam, Philadelphia: Benjamins, 319332. 
Sinclair, J. (1991). Corpus Concordance Collocation. Oxford: Oxford University Press.

Sinclair, J. (1996). «The Search for Units of Meaning», in TEXTUS, IX, 1: 75-106.

Singleton, D. (ed.) (1999). Exploring the Second Language Mental Lexicon. Cambridge: Cambridge University Press.

Shaughnessy, M. and J. Parkhill (2003). Culturally Authentic Pictorial Lexicon (CAPL). Accesible en: http://www.washjeff.edu/capl/ [Última consulta: 24.01.08].

Stubbs, M. (1995). «Collocations and semantic profiles. On the cause of the trouble with quantitative studies», in Functions of Language, 2, 1: 23-55.

Wittgenstein, L. (1953). Philosophical Investigations. Versión consultada: Philosophische Untersuchungen (1977). Frankfurt a. M.: Suhrkamp.

Wollert, M. (2002). Gleiche Wörter - andere Welten. Interkulturelle Vermittlungsprobleme im Grundwortschatzbereich. München: iudicium.

Wolter, B. (2001). «Comparing the L1 and L2 mental lexicon: A depth of individual word knowledge model», in Studies in Second Language Acquisition, 23: 41-69. 